Bettis Atomic Power Laboratory

P.O. Box 79

West Miffin, PA 15122-0079
Bechtel Bettis, Inc.

B-MT(SPME)-26

Manager, Pittsburgh Naval Reactors Office

\title{
FEB 232006
}

U. S. Department of Energy

Post Office Box 109

West Mifflin, Pennsylvania 15122-0109

Subject: $\quad$ Double Retort System for Materials Compatibility Testing (U)

Dear Sir:

This letter provides technical information regarding the assembly and operation of a double retort materials compatibility testing system and initial experimental results.

\section{Background}

With Naval Reactors (NR) approval of the Naval Reactors Prime Contractor Team (NRPCT) recommendation to develop a gas cooled reactor directly coupled to a Brayton power conversion system as the Space Nuclear Power Plant (SNPP) for Project Prometheus (References a and b) there was a need to investigate compatibility between the various materials to be used throughout the SNPP. Of particular interest was the transport of interstitial impurities from the nickel-base superalloys, which were leading candidates for most of the piping and turbine components to the refractory metal alloys planned for use in the reactor core. This kind of contamination has the potential to affect the lifetime of the core materials.

\section{Discussion}

There are a variety of systems to test compatibility between materials that are in direct or thermodynamic contact with each other. The easiest and least expensive system to use is the capsule testing in which two materials are put together inside a sealed capsule and heated to the operating temperature. Its main disadvantage is that it is isothermal, but its low cost and ability to run many samples simultaneously make it a useful research tool. On the other side of the cost/complexity spectrum is the forced circulation loop in which the gas transport medium is circulated throughout the system with a blower or turbine to mimic the Brayton cycle and there is independent temperature control for each of the materials being tested. The system described in this document is midway between these two other systems with respect to complexity and cost. It has independent temperature control on both materials being tested but it is a single pass design so that the inert gas used is not recirculated. In this arrangement a source material is placed in a retort which is immediately followed by a second retort with a sink material inside. Any gaseous impurity given off by the source material is transported by the inert gas to the sink material in the second retort where it could potentially react. A variety of materials compatibility experiments were planned before the SNPP program cancellation but only three experiments were performed. These three experiments were between a nickel based superalloy and a refractory metal with the purpose of testing compatibility between the core materials (refractory metals) and the balance of the SNPP, specifically high temperature turbine components. 


\section{Conclusions}

The use of a double retort system to test materials compatibility through the transfer of impurities from a source to a sink material is described here. The system has independent temperature control for both materials and is far less complex than closed loops. The system is described in detail and the results of three experiments are presented.

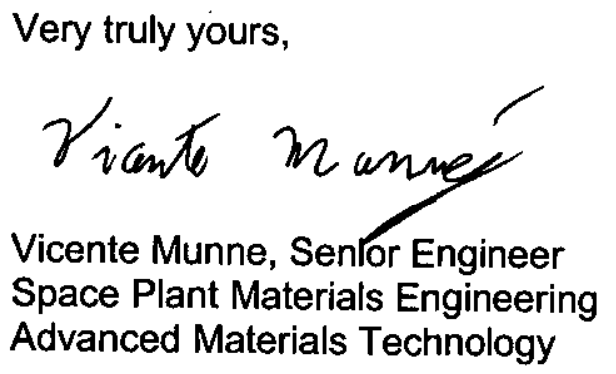

Approved by:

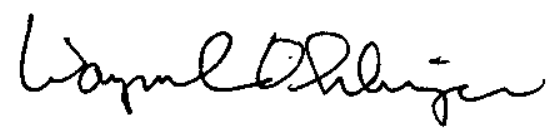

Wayne L. Ohlinger, Manager

Space Plant Materials Engineering

Advanced Materials Technology

\section{Attachments}

Attachment 1: Double Retort System Description (U)

Attachment 2: Double Retort System Experimental Runs (U)

\section{References}

(a) K. H. Donald, , "Space Nuclear Power - Reactor Coolant and Power Conversion System Concept - Approval Of, "NR Letter \#05-01228 dated 4/20/05

(b) D. R. Riley, "Space Nuclear Power Plant Concept Selection, For NR Approval," KAPL Letter SPP-67110-0005 / Bettis Letter B-SE-0077, dated 3/4/05 
Attachment 1 to

B-MT(SPME)-26

Page 1

Attachment 1

Double Retort System Description (U)

Author:

Vicente Munne

Reviewed by:

Wayne Ohlinger 
Attachment 1 to

B-MT(SPME)-26

Page 2

1. System Description: The system consists of two retorts in series. The first retort has a temperature capability of around $1350 \mathrm{~K}$ and the second one has a capability of $1400 \mathrm{~K}$. The first retort is where the material which is considered to be a potential source of impurities is located (source material). The second retort is where the material that might be affected by the impurities is located (sink material). Figure 1 shows a schematic of the system.

a. Layout: The inlet of the retort is connected to a turbo pump capable of pumping the system down to $2 \times 10^{-6}$ torr. The inlet gas comes from a manifold of three helium tanks that permit runs of slightly more than 100 hours. From the manifold the helium passes through the retorts, passes through an oxygen and water vapor sensor, and exits the system through a bubbler filled with diffusion pump fluid. A picture of the system is provided in Figure 2.

b. Retort Materials: Initially, the retort walls were made of a single 316 stainless steel tube. The leak rate of the retort system should not be higher than $5 \times 10^{-3}$ torr-lit/min. To avoid contamination coming from the wall of the $1400 \mathrm{~K}$ retort influencing the results of the experiment, a 12 inch long liner of mullite or alumina was located in that retort so that the helium gas would carry away downstream any material coming from the wall of the retort that might affect the refractory metal sample. This ceramic liner was supported by two semicircular alumina end caps to avoid direct contact with the metal retort walls.

c. Furnaces: Both the source and sink furnaces have three independently controlled zones. They have nichrome wire heating elements and work on $220 \mathrm{~V}$ single phase power.

d. Controls: Each furnace had three independent controllers so each of the three zones of the furnace could be set to a particular temperature. Each furnace has three type $K$ control thermocouples external to the retort. The external thermocouples are the control elements, but the setpoint is determined by internal thermocouples located in close proximity to the specimens. This means that the controllers are set to a temperature, offset from the desired specimen temperature, which will maintain the desired specimen temperature as indicated by the internal thermocouple.

e. Data acquisition: The data acquisition system is connected to four type $K$ thermocouples that are inside the retort. The first three thermocouples are on the source material side and roughly correspond to the three heat zones of the furnace. The large size of the source material sample required putting the three thermocouples in this location. The other thermocouple is in the sink material retort at the sample location. In addition, the system also monitored the outlet oxygen and water contents of the gas stream. After the first run, an oxygen monitor was also installed on the inlet side. 
Attachment 1 to

B-MT(SPME)-26

Page 3

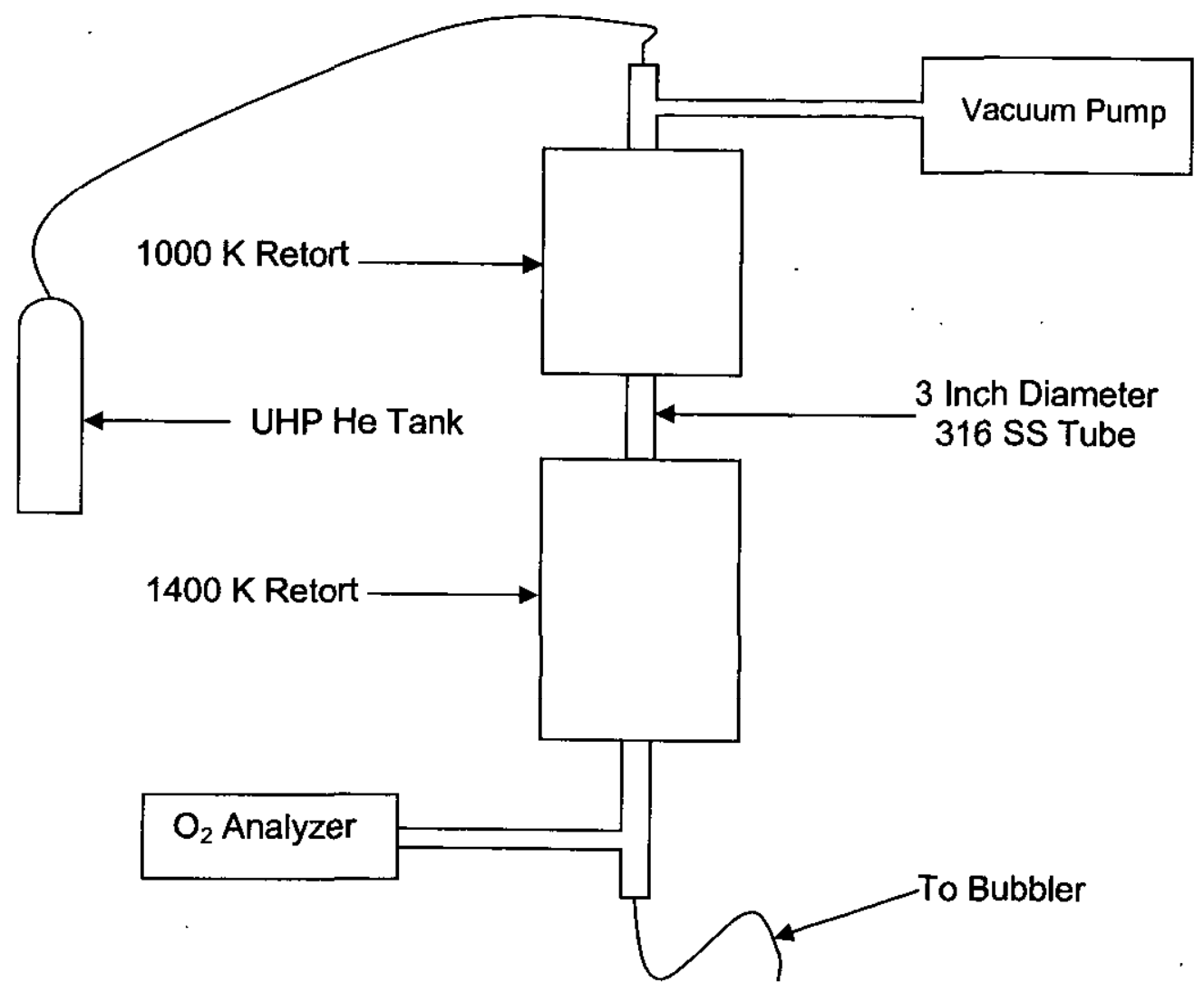

Figure 1: Schematic of Retort System for Materials Compatibility Tests 


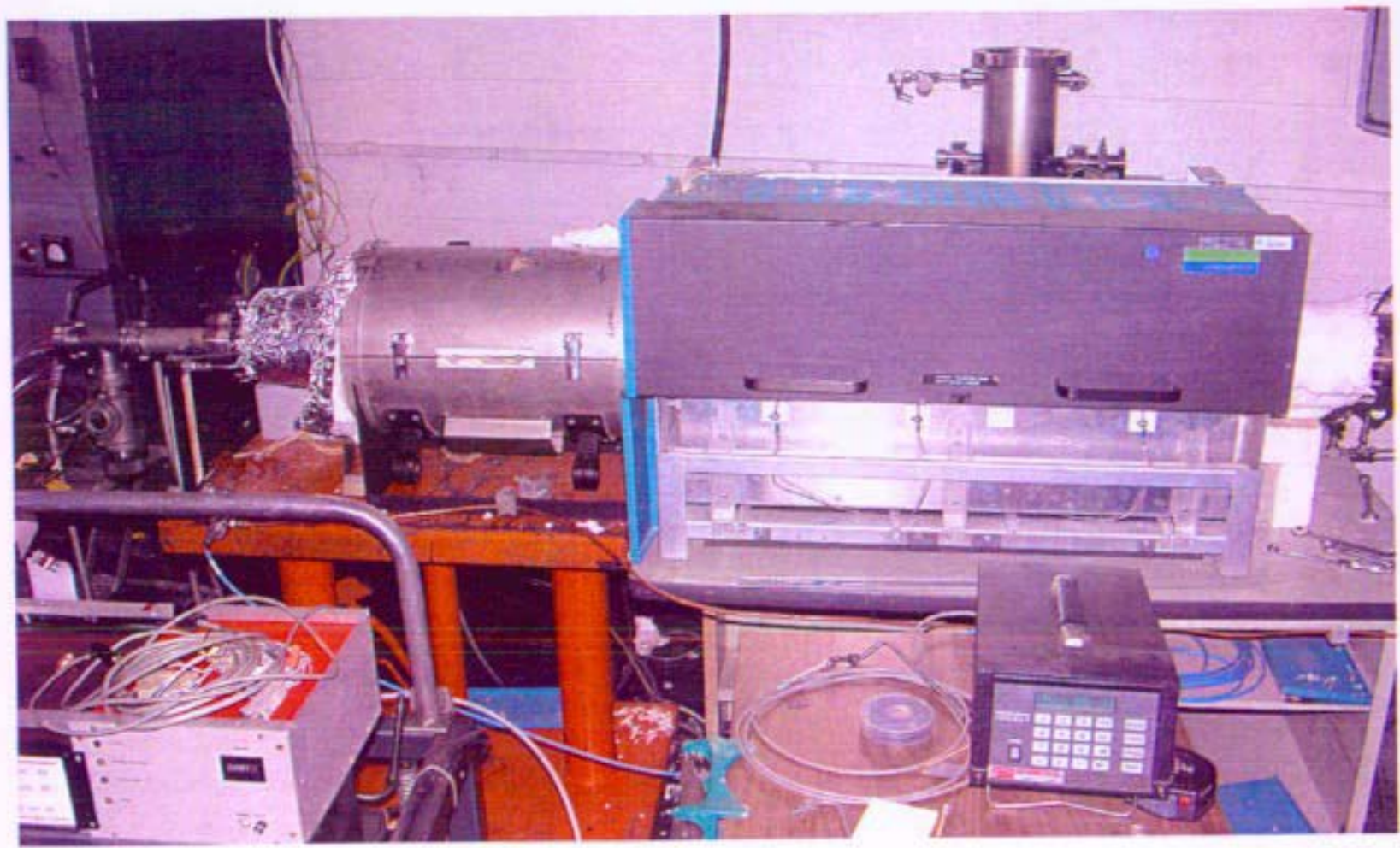

Figure 2: Double Retort System

2. Sample Preparation: The samples were designed with an area ratio roughly equivalent to the area ratios of the materials in the preconceptual SNPP design. For these experiments an area ratio of about 100 to 1 between the superalloy and the refractory alloy was selected.

a. Superalloy Sample: For the superalloy sample one of the notional superalloy materials was selected, Haynes 230. The sample was prepared out of 9 pieces of 0.040 " thick strip material. The strips were 15 inches in length and were held together with a $0.125^{\prime \prime}$ thick strip welded to the parts. Figure 3 shows a sketch with the widths and separations of the 9 strips. To avoid contact with the stainless tube, which might affect post experimental weight measurements on the samples, two thin strips of the same materials sized $0.25 \times 4$ inches, bent into a semicircle, were put at the front and the back of the sample arrangement between the samples and the stainless retort tube. Pictures of the assembled samples can be seen in Figure 4. In addition to this superalloy sample, a coupon of the same Haynes 230 material was inserted in each run to serve as a control piece for measurement of weight change. A picture of the coupon can be seen in Figure 5.

b. Refractory Alloy Sample: These samples were made of $0.025^{n}$ thick sheet material 1 inch $\times 3$ inches in size. The samples had a right angle bend at the bottom to make it self supporting and were located parallel to the gas flow. A sketch of these samples is shown in Figure 6. 
Attachment 1 to B-MT(SPME)-26

Page 5

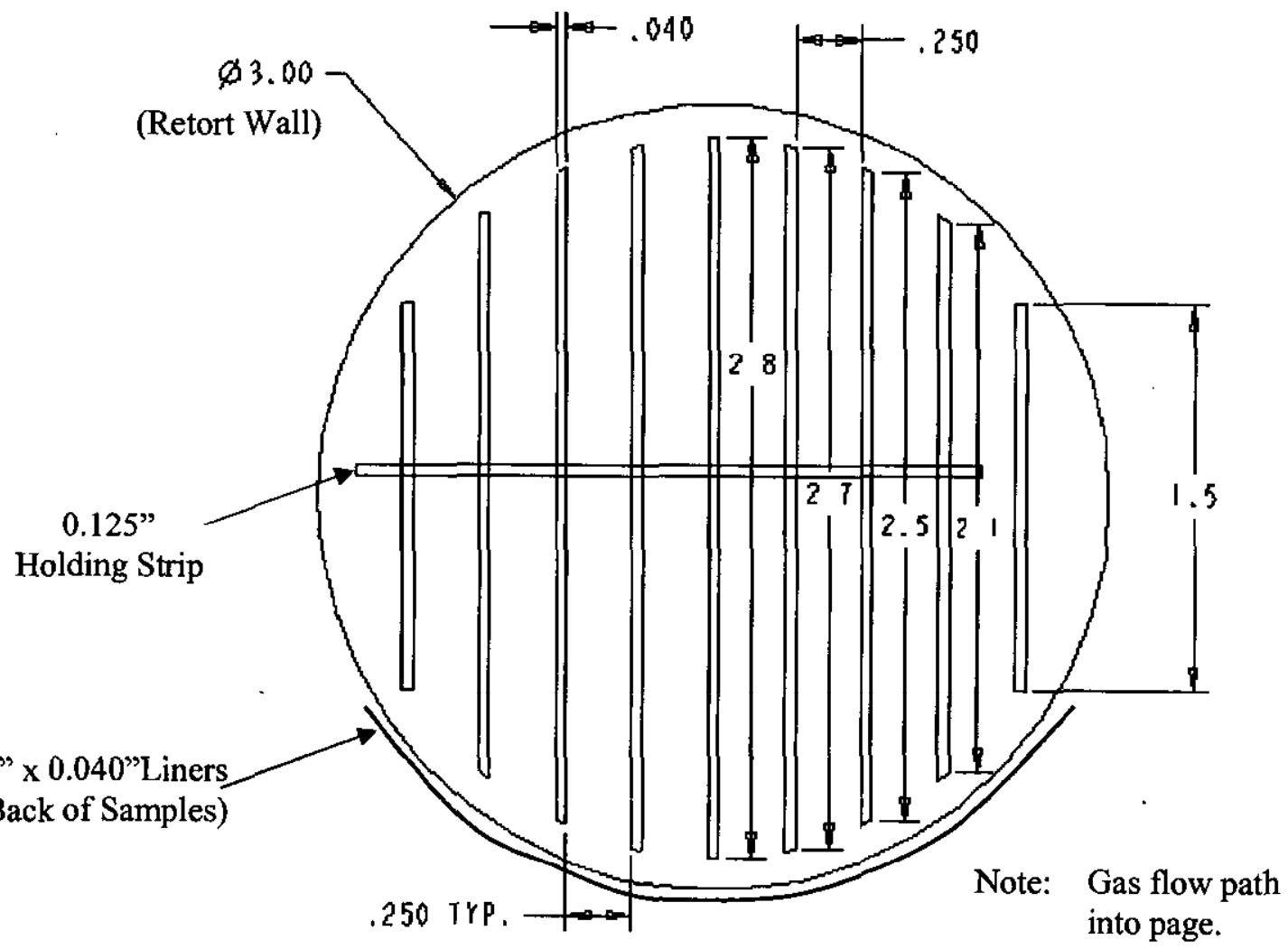

Figure 3: Geometry of Superalloy Loading on 1000K Retort 
Attachment 1 to

B-MT(SPME)-26

Page 6
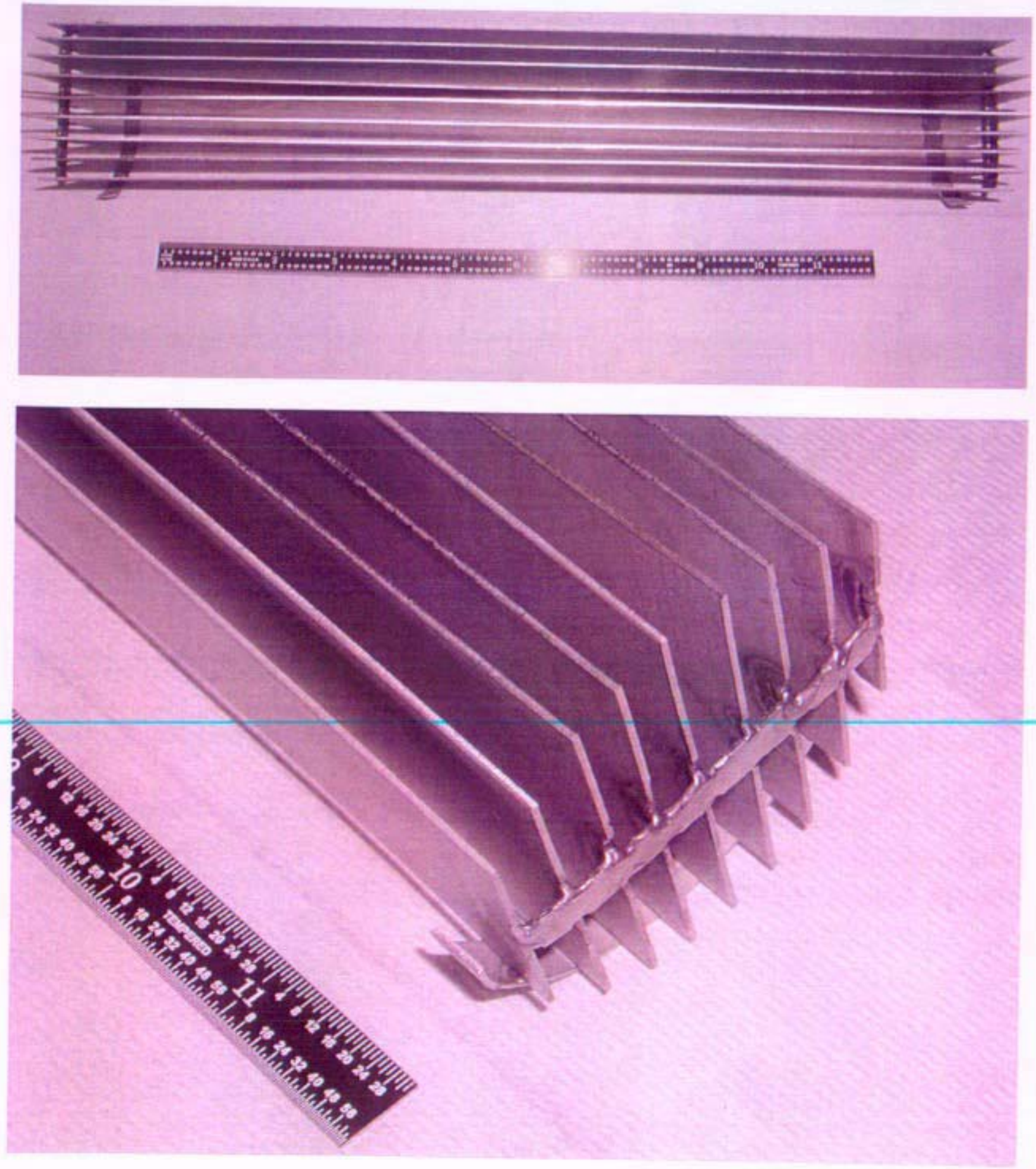

Figure 4: Various Views of the Superalloy Sample 
Attachment 1 to

B-MT(SPME)-26

Page 7

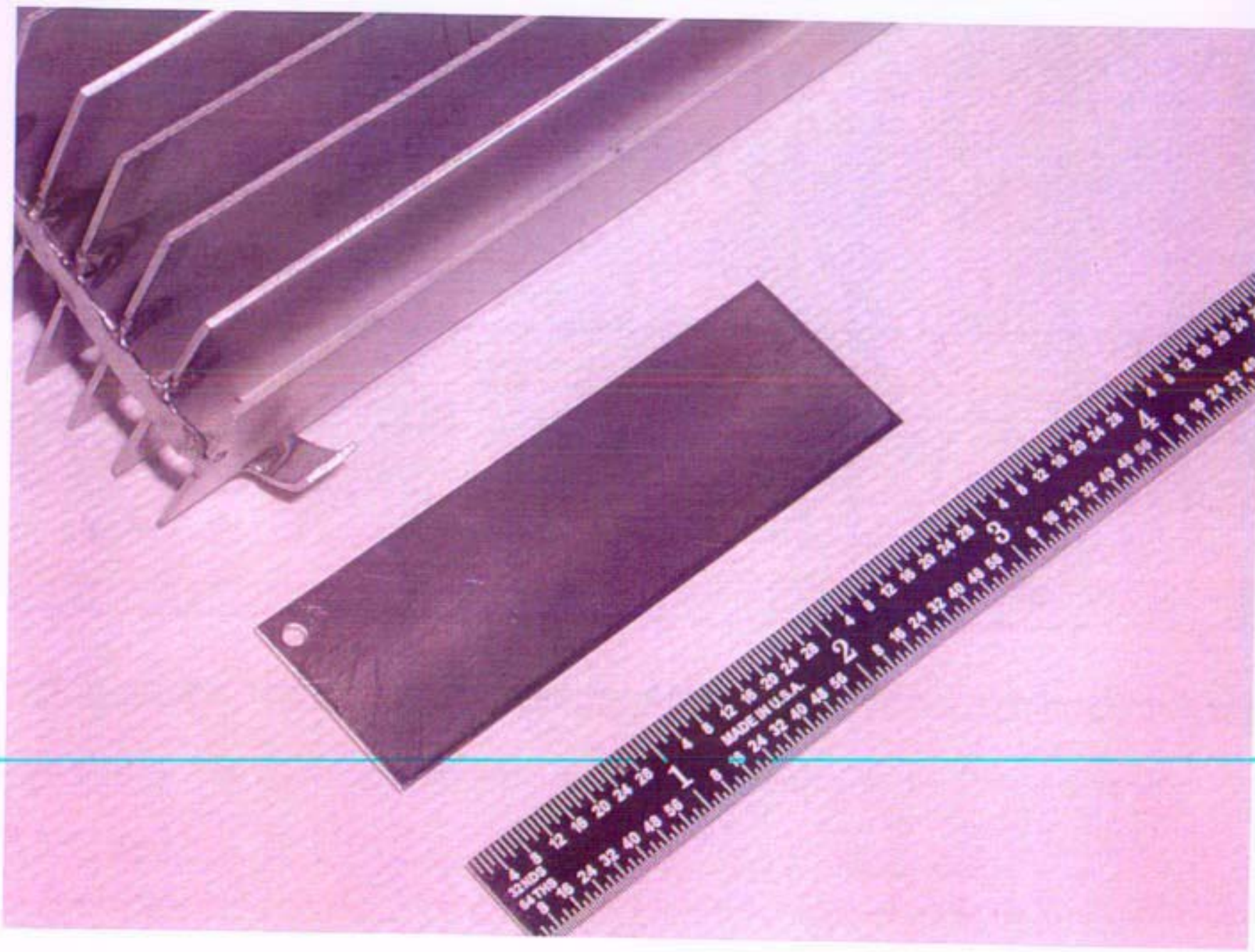

Figure 5: Superalloy Coupon Used for Weight Measurements 
Attachment 1 to

B-MT(SPME)-26

Page 8

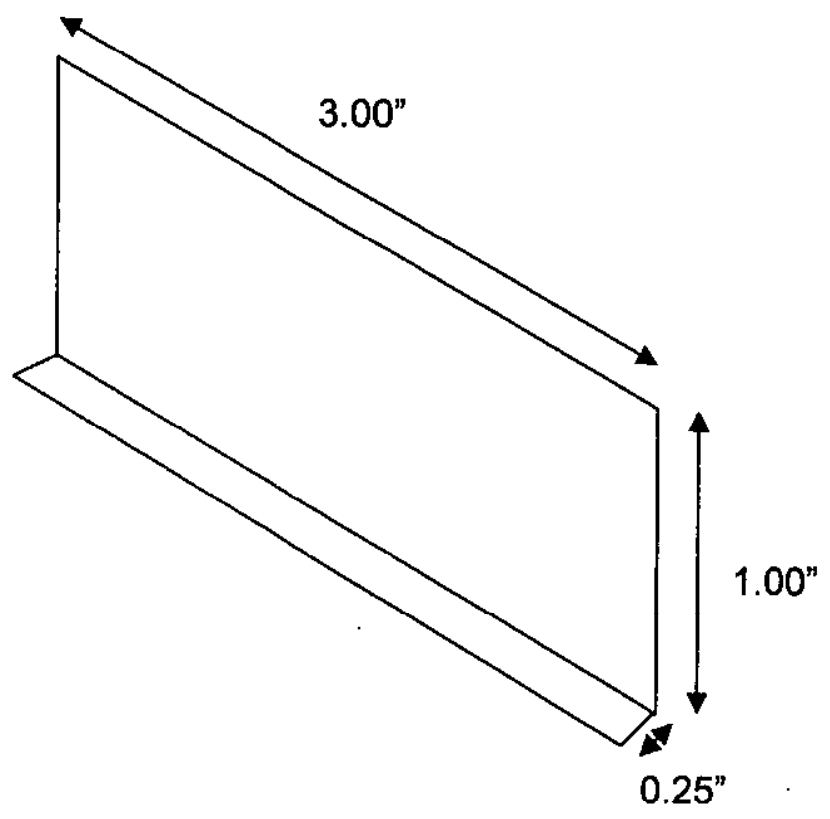

Figure 6: Geometry of Refractory Metal Samples 
Attachment 2 to

B-MT(SPME)-26

Page 1

Attachment 2

Double Retort System Experimental Runs (U)

Author:

Vicente Munne

Reviewed by:

Wayne Ohlinger 
Attachment 2 to

B-MT(SPME)-26

Page 2

1. Test Run Observations: Before termination of NRPCT participation in the Prometheus program an initial series of 10 experimental runs was planned but only three experiments were completed. The experiments were done according to the schedule shown on Table 1

Table 1: Run Schedule

\begin{tabular}{|c|c|c|c|c|}
\hline $\begin{array}{c}\text { Run } \\
\text { Number }\end{array}$ & $\begin{array}{c}\text { Duration } \\
\text { (hrs) }\end{array}$ & $\begin{array}{c}\text { Source Material } \\
\text { Temperature (K) }\end{array}$ & $\begin{array}{c}\text { Sink Material } \\
\text { Temperature (K) }\end{array}$ & Refractory Alloy \\
\hline 1 & 100 & 1000 & 1400 & $\mathrm{Nb}$ \\
\hline 2 & 100 & 1000 & 1400 & Ta-10W \\
\hline 3 & 100 & 1000 & 1400 & Mo-48Re \\
\hline
\end{tabular}

a. Pre-test procedure: The superalloy was acid dipped and cleaned with acetone before the first run. For subsequent runs the superalloy specimen was acetone cleaned only. The same Haynes 230 sample was used for the three runs. The refractory metal samples were also cleaned with acetone before the runs. Samples were loaded into their respective retorts and the system was closed up.

b. Start of run: The system was initially pumped down to a base pressure of $2 \times 10^{-6}$ Torr. After reaching that pressure the system was baked at $475 \mathrm{~K}$ for 24 hours and subsequently the run was started. The Helium flow rate was 1,100 SCCM. This flow rate was selected because this is the range where the oxygen and humidity sensors start operating reliably. The ramp up time to temperature was approximately 7 hours. The temperature controllers were ramped up manually until the setpoint was reached and then switched to automatic control.

c. Run: The run was monitored with the data acquisition system that printed process values every 15 minutes. The values were four temperature measurements, three points on the superalloy and one for the refractory metal specimen, as well as oxygen and water content at the outlet. The three temperature values of the superalloy source materials were maintained within $10 \mathrm{~K}$ of each other. After 100 hours the system was turned off and allowed to cool overnight before removing the samples.

d. Post run examination: After cooling, samples were removed from the retort and visually examined. The superalloy coupon and the refractory alloy sample were weighed to determine any mass change and the refractory alloy was examined in the metallograph and an inert gas fusion analysis of carbon, nitrogen, and oxygen was performed.

2. Experimental Runs: The three experimental runs done during this project are described as follows:

a. Niobium Sample: The first sample that was run was a pure niobium specimen. After sample loading, the start up procedure previously described was performed. Temperature was reached in approximately 7 hours and left at temperature for $100 \mathrm{hrs}$ as planned. After 100 hours at temperature the furnaces were shut down and the retorts were left to cool overnight with the helium gas flowing. Both the niobium sample and the Haynes 230 coupon were then removed and weighed to determine any mass changes during the run. The balance used for weighting had a precision of $1 \times 10^{-4}$ gram. It was found that the niobium metal had a weight gain of $0.208 \%$, while the superalloy showed a weight gain of $0.00797 \%(79.7 \mathrm{ppm})$. The oxygen content of the exiting helium gas varied from $5.1 \mathrm{ppm}$ at the start of the run to $2.3 \mathrm{ppm}$ at the end. The oxygen readings were relatively stable although readings as low as $1.9 \mathrm{ppm}$ were 
Attachment 2 to

B-MT(SPME)-26

Page 3

recorded intermittently toward the end of the run. Readings of water content started at $80.5 \mathrm{ppm}$ at the beginning of the run, dropping to $5.7 \mathrm{ppm}$ after 24 hours and $2.8 \mathrm{ppm}$ after 48 hours where the values stabilized and varied $\pm 0.3 \mathrm{ppm}$. The samples were also sent for determination of oxygen, nitrogen, and carbon concentration. Table 2 shows the analysis results

Table 2: Oxygen, Nitrogen, and Carbon Analysis of Niobium Samples

\begin{tabular}{|c|c|c|c|}
\hline Sample & Oxygen (ppm) & Nitrogen $(\mathbf{p p m})$ & Carbon $(\mathrm{ppm})$ \\
\hline As received & 94 & 1.5 & 15.5 \\
\hline After experiment & 288 & 173 & 54.5 \\
\hline
\end{tabular}

Discussion: Niobium reacts readily with interstitial species entrained in the gas stream upon release from the superalloy, i.e., oxygen and carbon. A weight gain of $0.208 \%$ is significant, indicating that the superalloy did react with materials carried by the helium gas. There was also a very small weight gain by the superalloy sample. That was unexpected because the superalloy should have given up interstitials and not absorbed any helium gas. This observation leads to the tentative conclusion that both materials reacted with residual gases in the helium atmosphere. The most likely source of these residual gases are impurities in the helium gas itself or leaks from the lines or other parts of the gas delivery system. To be able to determine the source of the impurities an additional oxygen sensor was installed in the inlet side to be used for the following runs. With the additional oxygen sensor it was possible to determine if the oxygen content in the helium gas came from inside the retort or from the helium cylinder and the gas lines. Also the high additional nitrogen content in the niobium sample also appeared to indicate that possibly there was an air leak in the system or that the helium gas was contaminated. A lot analysis of the helium indicated an oxygen content of $1.5 \mathrm{ppm}$ and a nitrogen content of $2.0 \mathrm{ppm}$ which would support the position that the reactive gases came from a leak.

b. Ta-10W Sample: The second sample (Ta-10W) was run with the same equipment and conditions but with an added oxygen sensor on the inlet side of the retorts to monitor the purity of the helium coming from the cylinders. The Haynes 230 superalloy sample was reused, but with a new unused control coupon attached to it. The refractory alloy sample was prepared with the same geometry as the niobium sample. After loading the samples and pumping the system down, heating of the retorts was initiated, but, before reaching temperature, the high temperature retort burned out a heating element and the run was terminated. A new furnace was ordered and, while removing the old one, it was found that the stainless tube was severly corroded. Scaling of oxide from the retort material might have contributed to the shorting of the heating element. A new Inconel tube was installed providing a higher operational temperature limit than the stainless steel. This tube was 4 inches in diameter instead of the stainless steel tube's 3 inches, but this should not have had any effect on the results of the experiments. With the new tube and furnace the system was leak checked and evacuated to restart the run. The restart proved difficult as the temperature did not stabilize. Upon further investigation it was discovered that the furnace was miswired so, after correcting the wiring, the run was restarted once again. The heating rate was identical to the previous run, reaching temperature in about 7 hours from when the run started. Initial readings of the sensors were inlet oxygen 9.3 
Attachment 2 to

B-MT(SPME)-26

Page 4

ppm, outlet oxygen $5.0 \mathrm{ppm}$, and outlet water $13.8 \mathrm{ppm}$. After 24 hours the readings were inlet oxygen $4.0 \mathrm{ppm}$, outlet oxygen $6.3 \mathrm{ppm}$, and outlet moisture $11.1 \mathrm{ppm}$. Oxygen values were then stable within $1 \mathrm{ppm}$. After about 55 hours into the run the temperature on the refractory alloy retort started drifting down and it was found that the middle heating element had failed so the run was terminated after 72 hours. It was later discovered that the furnace heating element was intact but rather a connector between the heating element and the electrical power wire had melted. Samples were weighed after removal from the system and a $0.0042 \%$ weight gain was found for the Haynes 230 and a $0.079 \%$ weight gain was found for the Ta-10W alloy. The samples were also sent for determination of oxygen, nitrogen, and carbon content. Table 3 shows the analysis results

Table 3: Oxygen, Nitrogen, and Carbon Analysis of Ta-10W Samples

\begin{tabular}{|c|c|c|c|}
\hline Sample & Oxygen $(\mathbf{p p m})$ & Nitrogen $(\mathrm{ppm})$ & Carbon $(\mathrm{ppm})$ \\
\hline As received & 94.2 & 2.6 & 35.5 \\
\hline After experiment & 262.1 & 3.5 & 77.9 \\
\hline
\end{tabular}

In addition to the numerical values obtained from the experiment it was noted that the alumina tube in the vicinity of the location of the Ta-10W displayed black spots (Figure 7). These spots are typical of a metallic reaction with the ceramic in an inert atmosphere. The spots appeared on the horizontal surfaces of the tube (outside top and inside bottom) with more spots on the outside of the tube than on the inside. This suggests that the particle size of the contamination was relatively large. It does not appear that the source of these particles was the Haynes 230 no evidence of similar contamintion was observed in the previous run. Nevertheless, to investigate the source of the contamination, the tube was analyzed using $x$-ray fluorescence and it was found that the spots contained iron, nickel, and chrome which were not present on the uncontaminted (white) surface of the alumina tube. These contaminants indicate that the most probable contamination material is stainless steel. Given that the particle were relatively large and that the flow rate that was used $(1,100$ SCCM) is not sufficient to transport such a large amount of particles large distances, the most plausible explanation would be that the metallic particle transport was a single event that occurred when the system was backfilled with helium after being under vacuum. The most likely source of the contamination is swaging or machining residue from any of the lines or flanges that were installed when the stainless steel tube was exchanged for the Inconel retort. Although the source of the metallic contamination can not be confirmed at this time it is highly unlikely that it came from the Haynes 230 sample given the morphology and composition of the contamination. 


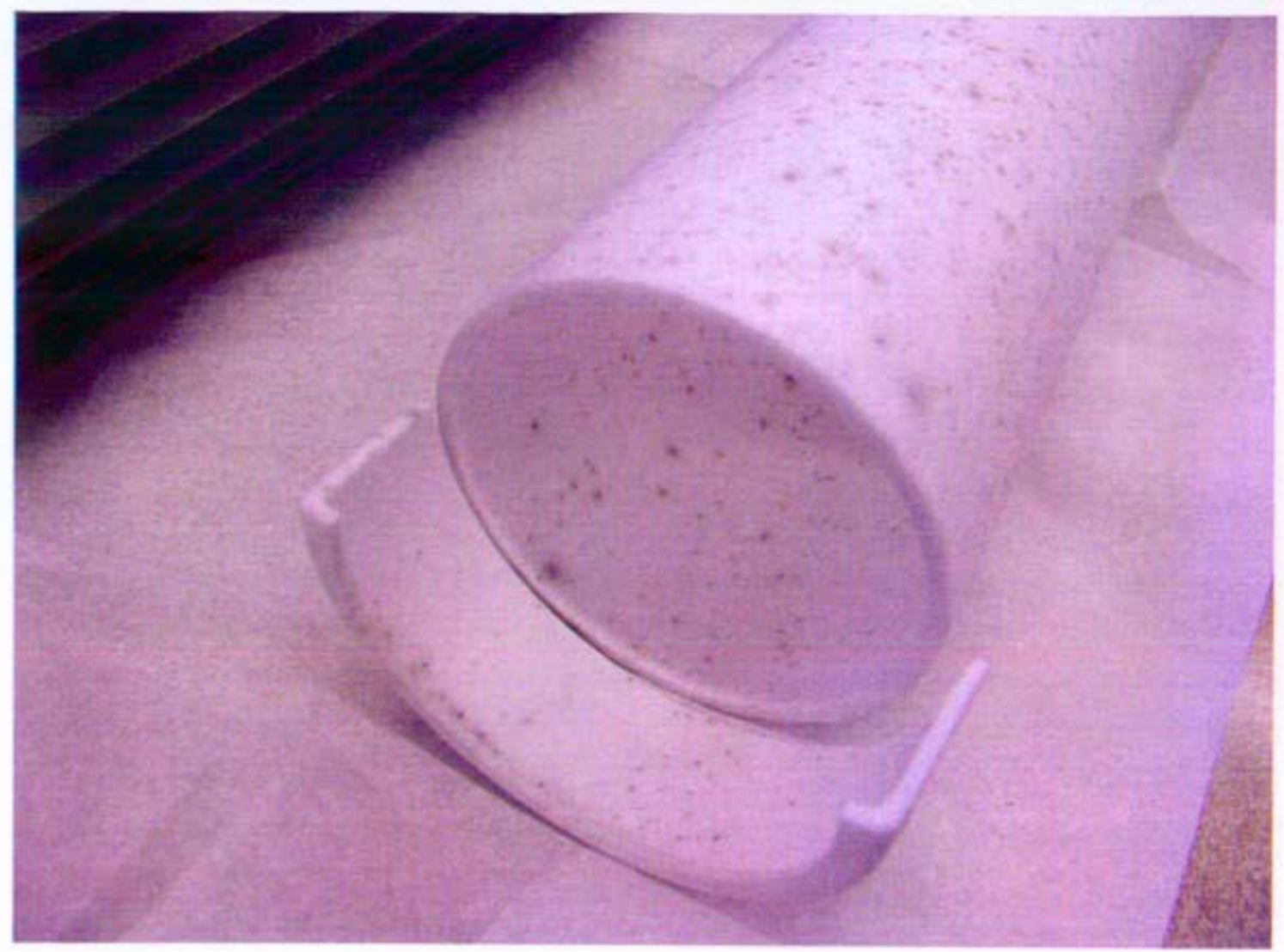

Figure 7: Contamination Found on Alumina Tube after Ta-10W Run

Discussion: Ta-10W, although less reactive than niobium, was also expected to react with any interstitials coming from the superalloy. The weight gain observed in the superalloy of $0.0042 \%$ was about half of the previous run which seems to confirm the presence of a leak in the previous run or at least a larger leak than in the second run. The addition of the new oxygen sensor at the retort gas inlet showed that the inlet concentration of oxygen was slightly higher than the outlet which is expected as some of the oxygen would react with the samples in the retorts. The weight gain of the refractory alloy was smaller than in the case of the niobium which was expected given the lower reactivity of this alloy compared with the niobium and also because the smaller weight gain of the Haynes 230 indicates a reduced presence of reactive gases.

c. Mo-47.5Re Sample: The last experiment run was a Mo-Re alloy. This alloy is the most stable of the three materials used and it was expected to show the least amount of reaction with the typical interstitial contaminants. After sample loading the start up procedure was followed in the same way as the previous two runs. For this run the temperature of the second retort was decreased to $1300 \mathrm{~K}$ to improve the reliability of the system. At this temperature the effects of interstitials on the refractory metal alloy were still expected to be noticeable. Additionally, the contaminated alumina tube from the previous experiment was not reused but substituted with a mullite tube. After startup, temperature was again reached in approximately 7 hours and left at temperature for $100 \mathrm{hrs}$ as planned. After 100 hours at temperature the furnaces were shut down and the retorts were left to cool overnight with the helium gas flowing. The 
inlet oxygen content of the helium gas varied from 0.9 to $2.0 \mathrm{ppm}$ staying near $1.0 \mathrm{ppm}$ for most of the run. The outlet oxygen sensor varied around $4.1 \pm 0.5 \mathrm{ppm}$ for most of the run. These values were very stable throughout the run. The moisture sensor started at $52 \mathrm{ppm}$ and stabilized at $6.5 \mathrm{ppm}$ after 24 hours where the values remained stable varying only $\pm 1 \mathrm{ppm}$. Weight measurements for the Haynes 230 indicated a $0.0042 \%$ weight gain while the Mo-47.5Re sample showed a weight loss of $-0.0014 \%$. The samples were also sent for determination of oxygen, nitrogen, and carbon content. Table 4 shows the analysis results

Table 4: Oxygen, Nitrogen, and Carbon Analysis of Mo-47.5Re Samples

\begin{tabular}{|c|c|c|c|}
\hline Sample & Oxygen $(\mathbf{p p m})$ & Nitrogen $(\mathbf{p p m})$ & Carbon $(\mathrm{ppm})$ \\
\hline As received & 31.1 & 3.0 & 16.2 \\
\hline After experiment & 31.8 & 1.9 & 31.3 \\
\hline
\end{tabular}

Discussion: Mo-47.5Re is the least reactive of the alloys studied. The chemical analysis shows that it gained very little oxygen, lost some nitrogen and gained carbon. The fact that a weight loss was observed instead of a weight gain is additional indication of the lower reactivity of this alloy. The stable and low oxygen readings obtained throughout the run indicate that the system itself was well outgassed. The new mullite tube did show some change of color but not the previously observed spots. The part that was closest to the metal retort tube (at the bottom) showed the most darkening (Figure 8). This darkening is probably a reaction with a material evaporating from the retort walls.

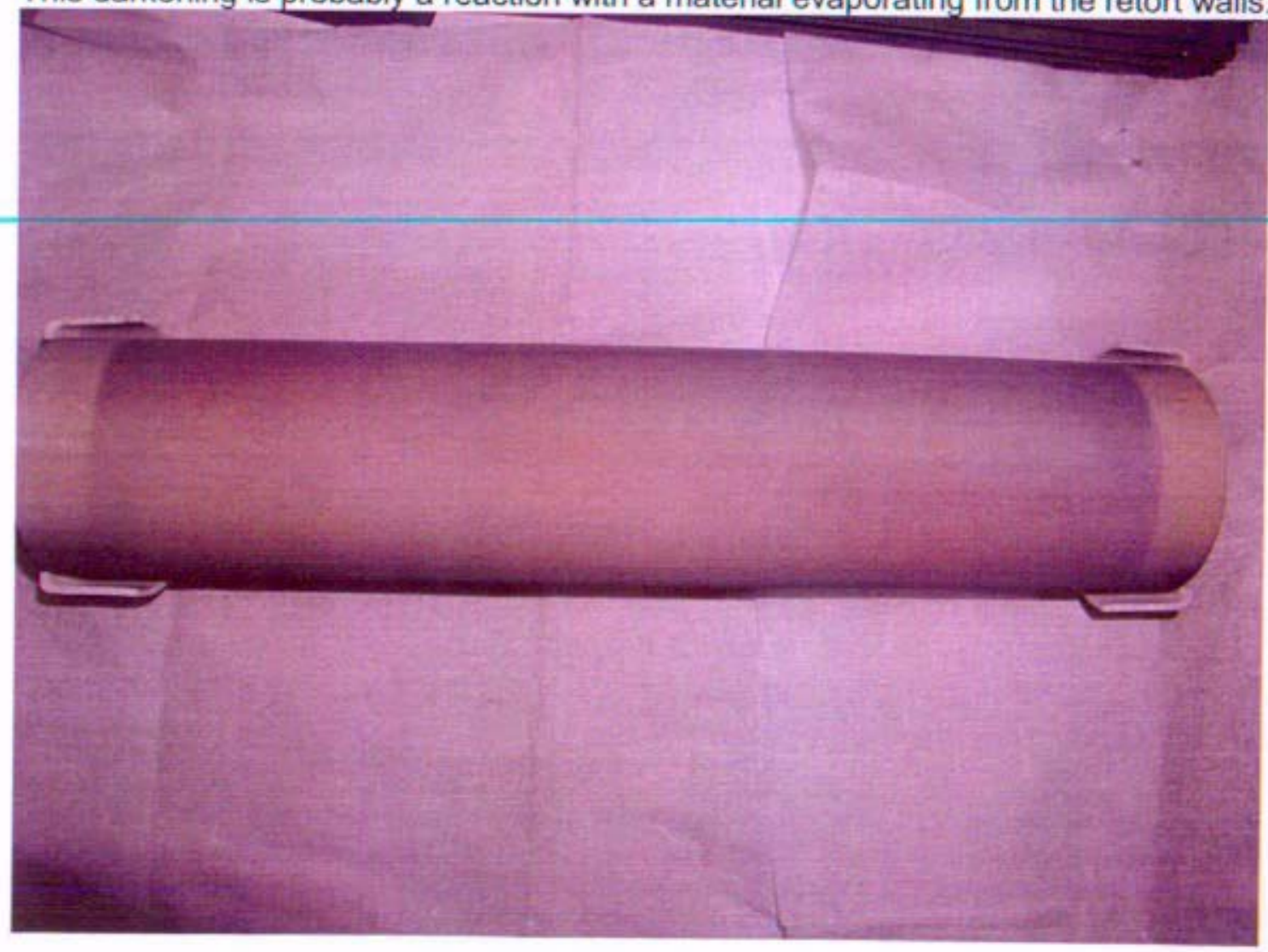

Figure 8: Discoloration Observed on Mullite Tube after Mo-47.5 Re Run 
Attachment 2 to

B-MT(SPME)-26

Page 7

The weight gain of the Mo-Re alloy was smaller than in the case of the niobium which was expected given the lower reactivity of this alloy compared with the niobium and also because the smaller weight gain of the Haynes 230 indicates a smaller presence of reactive gases.

3. Conclusions and Recommendations: The results obtained from these experiments indicate that this system does have the potential to be used for materials compatibility testing but there is a need for more development to optimize the system. Given that the SNPP project was terminated after only the first experiment was completed, there was not enough time and funding to further optimize the facilities. With more time and resources the following steps should be taken:

a. Helium gas purity: The helium gas used for these test was UHP quality but the amount of oxygen should be reduced even further to avoid any oxygen from the helium reacting with the sample. It is recommended to find a higher purity helium source and to add a titanium gettering system which can be placed just before the helium gas inlet.

b. Furnaces: The furnaces used in these experiments used heating elements made of Nichrome wires. The temperatures used during the runs were close to the maximum allowed for the elements which decreased the reliability of the system, as was observed with the various electrical problems encountered. A furnace with silicon carbide or platinum heating elements, particularly in the high temperature retort, would be recommended.

c. Retort materials: The first retort was made of stainless steel which oxidized at the temperature reached in the high temperature section. The second retort material (Inconel) was stable at the operating temperatures but had apparent contamination problems. The contamination problems could be because the tube was previously used in other experiments. However, it is also a potential problem that the tube itself may release interstitials that coould interfere with the experimental results. To minimize this problem it is recommended to use a more thermodynamically stable material like alumina, mullite, or fused silica glass for the retort material.

d. Qualification of the system: To determine if the effect of interstitials from the source material retort can be reliably observed, a series of runs using only a refractory metal should be performed until it is confirmed that there is no change in the refractory metal impurity levels. In this work, given the time constraints forced by termination of the program, a single calibration run was done which was not enough to conclusively qualify the system. It is recommended to perform a number of runs using a highly reactive refractory metal (like niobium) and no superalloy to demonstrate the repeatability and cleanliness of the system. 
Attachment 2 to

B-MT(SPME)-26

Page 8

(This Page Intentionally Blank) 


\section{CONCURRENCE RECORD SHEET}

- DOCUMENT NUMBER: B-MT(SPME)-26

- THIS DOCUMENT CONTAINS INFORMATION WHICH SHOULD BE CONSIDERED FOR PATENT DISCLOSURES

- THIS DOCUMENT CONTAINS INFORMATION WHICH MEETS BETTIS WORK CATEGORIES [A,B,C,D or (N/A)]

CONCURRENCE SIGNATURES (Activity must be included) - RESOLVE COMMENTS BEFORE SIGNING

SIGNATURE/ACTIVITY

DATE

TYPE

DETAILS OF REVIEW REQUESTED (if necessary)

TYPE OF REVIEWS (to be determined by author) [See table at end of instructions for definitions.]

1-Peer:Summary 2-Peer:Intermediate 3-Peer:Detail 4-Independent 5-Informal Committee NAME TYPED AND SIGNATURE, OF NEXT HIGHER MANAGER NOT SIGNING ON LETTER tom 2 . parke

J. Hack, Manager, Advanced Materials Technology

- CONTRIBUTORS AND IMPACTED PARTIES NOT REQUESTED TO CONCUR AND WHY:

\section{DISTRIBUTION}

\section{Bettis}

M. N. Smith 02B/GM

S. D. Harkness, 01Q/MT

RC Jewart, 01C/SE

JE Hack, 05R/MT

M. Zika, 01C/SE

R. Baranwal, 05P/MT

D. P. Hagerty, 62M/LOS

W. L. Ohlinger, 05R/MT

R. W. Buckman, 05R/MT

G. M. Brewer, 38D/ADD

E. V. Carelli, 34N/ADD

V. Munne, 36E/NRF

C. D. Eshelman, 36E/ADD
NR

DI Curtis(3), 08S (INFO)

JD Yoxtheimer, 08S/8034

S. Bell, 08I/8024

DE Dei, 08A8011

WE Evers, 08E/8019

TJ Mueller, 08R/8033

JP Mosquera, 08C/8017

MD Natale, 08I/8024

TN Rodeheaver, 081/8024

SJ Rodgers, 08E/8019

$\mathrm{CH}$ Oosterman, 08C/8017

SJ Trautman, 08V/8037
KAPL

Y Ballout, 92

CF Dempsey, 111

DF McCoy, 111

SA Simonson, 081

MJ Woliman, 111

T. Schumaker, 103A

BPMI-P

SD Gazarik

RF Hanson

PNR

J. Andes

JF Koury

\section{SNR}

D. Clapper, 065

GM Millis, 065 